\title{
Structural characterization of clinically reported missense mutations identified in BRCA1
}

\author{
Neha Mishra', 2 , Suchita Dubey ${ }^{1,2}$ Ashok K Varma ${ }^{1,2}$ \\ ${ }^{1}$ Advanced centre for Treatment, Research and Education in cancer, Kharghar, Navi Mumbai, Maharashtra - 410210, INDIA, \\ ${ }^{2}$ Homi Bhabha National Institute, Training School Complex, Anushaktinagar, Mumbai, Maharashtra - 400094, INDIA
}

nmishra@actrec.gov.in

Germline loss of function mutations in Breast Cancer Susceptibility gene 1 (BRCA1) and Breast Cancer Susceptibility gene 2 (BRCA2) are known to be responsible for Hereditary Breast and Ovarian Cancer Syndrome (HBOCS). BRCA1 encodes 1863 amino acids consisting of N-terminus RING domain, intrinsically disordered central DNA binding region and highly conserved two tandem repeats of BRCTs constituting the C-terminus domain (CTD)[1]. The RING domain forms a heterodimer with BRCA1-associated RING domain protein 1 (BARD1) and acts as E3- Ubiquitin ligase. However, C-terminal of the BRCA1 is known to interact with proteins containing the consensus sequences of $\mathrm{pS}-\mathrm{X}-\mathrm{X}-\mathrm{F}$ motif to mediate different complex formation at the time of Double Strand Break Repair (DSBR). Majority of the missense mutations are found in the BRCT, RING domain and few in the central domain of BRCA1. Several studies have been performed to classify such variants of uncertain significance (VUS) in BRCA1 as pathogenic or neutral but the exact molecular mechanism of pathogenicity still remains to be deciphered[2,3]. The aim of the present study is to evaluate the structural significance of these missense mutations located in the central and C-terminus functional domains of BRCA1 using biophysical, biochemical and in-silico tools. It was found that BRCA1 Arg866Cys in the central region, Thr1691Arg and Gly1801Asp in the BRCT domain show conformational alterations. The central non-specific DNA binding domain has also been evaluated for its conformational changes in the presence and absence of super-coiled DNA. However, a reduced DNA binding ability was observed for the mutant as compared to the wild-type protein. Further, the central region of BRCA1 has been assessed for its intrinsically disordered behaviour. Addition of 2,2,2-trifluoroethanol (TFE) led to gain of structure of the central region and therefore, less susceptibility towards proteolysis. The mutations have been characterized with the help of Size exclusion chromatography (SEC), Circular Dichroism spectroscopy, nano DSF, EMSA. With this information we would further extend our studies for protein-protein interactions of the wild type and mutant proteins using ITC, SPR and co-crystallization. The reported results will enhance our understanding towards the fundamental structural differences arising due to cancer predisposing missense mutations.

[1] R. Roy, J. Chun, and S. N. Powell, "BRCA1 and BRCA2 : different roles in a common pathway of genome protection," Nat. Rev. Cancer.

[2] R. W. Anantha et al., "Functional and mutational landscapes of BRCA1 for homology-directed repair and therapy resistance," pp. 1-21, 2017.

[3] P. Bouwman, H. Van Der Gulden, I. Van Der Heijden, R. Drost, and C. N. Klijn, "RESEARCH ARTICLE A High-Throughput Functional Complementation Assay for Classifi cation of BRCA1 Missense Variants,” 2013.

\section{Keywords: Hereditary Breast Cancer; BRCA1; secondary structural changes; BRCT; Intrinsically disordered protein regions (IDPRs); DNA binding regions}

Acknowledgement-Funding for this study was supported by Annual Scientific Fund from ACTREC-TMC. The authors thank the XRD facility at ACTREC for providing necessary support to this study. 